\title{
A note on proving the strong NP-hardness of some scheduling problems with start time dependent job processing times
}

\author{
Radosław Rudek
}

Received: 4 February 2011 / Accepted: 9 April 2011 / Published online: 27 April 2011

(C) The Author(s) 2011. This article is published with open access at Springerlink.com

\begin{abstract}
In this paper, we show that the strong NP-hardness proofs of some scheduling problems with start time dependent job processing times presented in Gawiejnowicz (Eur J Oper Res 180:472-478, 2007) and Zhao and Tang (Optim Lett 5:183-190, 2011) are incorrect. Namely, the applied transformations from 4-PRODUCT problem to the considered scheduling problems are polynomial not pseudopolynomial. Thus, the related problems are NP-hard, but their complete computational status is still an open issue: ordinary or strongly NP-hard?
\end{abstract}

Keywords Complexity theory $\cdot$ Scheduling $\cdot$ Deteriorating jobs $\cdot$ Makespan

\section{Introduction}

Gawiejnowicz [2] and Zhao and Tang [5] claimed to prove the strong NP-hardness of some scheduling problems with start time dependent job processing times on the basis of 4-PRODUCT problem. In this note, we show that the proofs are incorrect, since the authors made the same mistake. Namely, instead of a pseudopolynomial time transformation from 4-PRODUCT, they applied the polynomial one. Although the authors proved NP-hardness, however, the scheduling problems are still unknown to be ordinary or strongly NP-hard. Moreover, we point out the main reason of these mistakes that is related with the definition of 4-PRODUCT.

R. Rudek $(\varangle)$

Wrocław University of Economics, Komandorska 118/120,

53-345 Wrocław, Poland

e-mail: radoslaw.rudek@ue.wroc.pl 


\section{Definition of 4-ProDuCT}

Following Gawiejnowicz [2] the definition of 4-PRODUCT problem (see [3] for details) is given.

4-Product (4-P): given $D \in Q^{+}$, a set $N=\{1,2, \ldots, 4 p\}$ for some natural $p$, and a size $D^{\frac{1}{5}}<y_{j}<D^{\frac{1}{3}} \in Q^{+}$for each $j \in N ; \prod_{j \in N} y_{j}=D^{p}$, do there exist disjoint subsets $\left\{N_{1}, N_{2}, \ldots, N_{p}\right\}$ such that $\bigcup_{i=1}^{p} N_{i}=N$ and $\prod_{j \in N_{i}} y_{j}=D$ for $1 \leq i \leq p ?$

Assuming that 4-P is strongly NP-complete [3], the above definition can cause misreading, since it suggests that $D^{p}$ is an input value of the problem. However, it is redundant since the problem can be defined without this number, thereby it only artificially increases the input size of the problem. In fact, the maximum value of the problem is $D$. This seemingly unimportant value suggests that the maximum value of 4-P can be bounded by $D^{p}$. Nevertheless, it was a crucial assumption in the proofs of the strong NP-hardness of some scheduling problems with start time dependent job processing times considered in [2] and [5]. Since the maximum value of 4-P is $D$, then instead of pseudopolynomial time transformations Gawiejnowicz [2] and Zhao and Tang [5] provided polynomial transformations, thereby, they did not prove the strong NP-hardness of the analysed problems.

\section{Pseudopolynomial time transformation}

In the further part of this note, we will show in details that the proofs given by Gawiejnowicz [2] are incorrect. Since the similar mistake was made by Zhao and Tang [5], but later and on the basis of the same definition of 4-P, then we will discuss it briefly.

\subsection{Gawiejnowicz (2007)}

Two of the main results presented in [2] are the proofs of the strong NP-hardness of the single machine makespan minimization problems with start time dependent job processing times and non-availability periods (TDSNP) and with ready times and deadlines (TDSRTD). These problems are defined as follows; we keep the terminology and notation used by the author.

There is given a set of independent, nonpreemptable and deteriorating jobs $J_{1}, J_{2}$, $\ldots, J_{n}$ to be processed on a single machine, starting from some time $t_{0}>0$. The processing time $p_{j}$ of job $J_{j}$ is a proportional function of the starting time $S_{j}$ of this job, $p_{j}=\alpha_{j} S_{j}$, where $S_{j} \geq t_{0}$ and deterioration rate $\alpha_{j}>0$ for $1 \leq j \leq n$. Because the starting time $S_{j}$ is the variable on which the processing time $p_{j}$ depends, for simplicity of presentation we will write $t$ instead of $S_{j}$. Moreover, since each job is completely defined by its deterioration rate, we will also identify job $J_{j}$ with its deterioration rate $\alpha_{j}$.

In the first problem (TDSNP), the machine is not continuously available and there are given $k<n$ disjoint periods of the machine non-availability. These periods are described by time intervals $\left[W_{i, 1}, W_{i, 2}\right]$, where $W_{1,1}>t_{0}$ and $W_{i, 1}<W_{i, 2}$ for 
$1 \leq i \leq k$. Moreover, the jobs are nonresumable, i.e. if a job has been interrupted by the start time of a non-availability period, then this job has to be restarted after the machine becomes available again.

In the second problem (TDSRTD), the machine is continuously available but for each job ready time $r_{j}$ and deadline $d_{j}, 1 \leq j \leq n$, are defined.

For both problems the objective is to find such a schedule that minimizes the maximum completion time $C_{\max }=\max _{1 \leq j \leq n}\left\{C_{[j]}\right\}$, where $C_{[j]}$ denotes the completion time of the $j$ th job in the schedule.

To prove the strong NP-hardness of TDSNP (Theorem 2) and of TDSRTD (Theorem 4), the author transform 4-PRODUCT problem [3], that is known to be NP-complete in the strong sense, to the decision versions of the considered scheduling problems. Their decision versions are defined as follows.

Time-dependent scheduling with non-availability periods (TDSNP) given numbers $t_{0}>0$ and $G_{1}>0$, a set $J=\{1,2, \ldots, n\}$ for some natural $n$, a number $\alpha_{j}>0$ for each $j \in J$ and numbers $W_{i, 1}<W_{i, 2}$ such that $t_{0}<W_{1,1}$ and $W_{i, 1}<W_{i, 2}$ for $1 \leq i \leq k<n$ for some $k \in Z^{+}$, does there exist a single-machine schedule for jobs with time-dependent processing times in the form of $p_{j}=\alpha_{j} t, 1 \leq j \leq n$, such that these jobs are scheduled starting from time $t_{0}$, the machine is not available for processing during time periods $\left[W_{i, 1}, W_{i, 2}\right], 1 \leq i \leq k$, and the maximum completion time for this schedule is not greater than $G_{1}$ ?

Time-dependent scheduling with ready times and deadlines (TDSRTD) given numbers $t_{0}>0$ and $G_{2}>0$, a set $J=\{1,2, \ldots, n\}$ for some natural $n$, numbers $\alpha_{j}>0, r_{j}>0$ and $d_{j}>0$ for each $j \in J$, does there exist a single-machine schedule for jobs with time-dependent processing times in the form of $p_{j}=\alpha_{j} t, 1 \leq j \leq n$, such that these jobs are scheduled starting from time $t_{0}$, the completion time $C_{[j]}$ of the $j$ th job in the schedule satisfies inequality $r_{[j]} \leq C_{[j]} \leq d_{[j]}, 1 \leq j \leq n$, and the maximum completion time for this schedule is not greater than $G_{2}$ ?

At first, we consider the proof of the strong NP-hardness of TDSNP (Theorem 2). The author used the following transformation from the 4-P problem: $n=4 p, t_{0}=1$, $\alpha_{j}=y_{j}-1$ for $1 \leq j \leq n, k=p, W_{i, 1}=\sum_{j=1}^{i} D^{j}, W_{i, 2}=\sum_{j=0}^{i} D^{j}$ for $1 \leq i \leq k$ and $G_{1}^{\prime \prime}=\sum_{j=1}^{p} D^{j}$.

Recall now a condition from a definition of a pseudopolynomial transformation $([1,4])$. Let $\pi_{1}$ and $\pi_{2}$ are two decision problems. Let $I_{\pi_{1}}$ and $I_{\pi_{2}}$ denote their sets of all possible instances, $\operatorname{Max}(I)$ denotes the maximum value for an instance $I$ and $N(I)$ is the size of $I$. Let $f: I_{\pi_{2}} \longrightarrow I_{\pi_{1}}$ denote the transformation from $\pi_{2}$ to $\pi_{1}$. One of the requirements for $f$ to be pseudopolynomial is such that there must exist a polynomial $q$ of two variables that holds:

$$
\forall I \in I_{\pi_{2}}: \operatorname{Max}(f(I)) \leq q(\operatorname{Max}(I), N(I))
$$

It means that the values of any instance $I$ of the problem $\pi_{2}$ cannot increase in an exponential manner if $\pi_{2}$ is transformed to $\pi_{1}$. 
Let $\pi_{2}$ denote $4-\mathrm{P}$ and $\pi_{1}$ is the considered scheduling problem. It is obvious that for the given transformation and $I \in I_{\pi_{2}}$, we have $\operatorname{Max}(I)=D, N(I)=4 p$, $\operatorname{Max}(f(I))>D^{p}$ and $N(f(I))=4 p$. Thus, there does not exist such $q$ for which (1) holds, thereby the transformation cannot be pseudopolynomial. Therefore, the proof of the strong NP-hardness (Theorem 2) is incorrect. Although Gawiejnowicz [2] did not prove the strong NP-hardness of TDSNP, he showed that the problem is at least NP-hard, but it is already covered by Theorem 1 of the related paper.

The same mistake was made for TDSRTD (Theorem 4) since the transformation from 4-P to the decision version of TDSRTD was given as follows: $n=5 p, t_{0}=1$, $\alpha_{j}=x_{j}-1, r_{j}=t_{0}, d_{j}=G_{2}^{\prime \prime}$ for $1 \leq j \leq 4 p, \alpha_{4 p+i}=D-1, r_{4 p+i}=D^{2 i-1}$ and $d_{4 p+i}=D^{2 i}$ for $1 \leq i \leq p$, where threshold $G_{2}^{\prime \prime}=D^{2 p}$. Therefore, the maximum values of the constructed instances of TDSRTD increase in an exponential manner depending on $p$, thus, there does not exist such $q$ for which (1) holds. Thereby, only NP-hardness is proved, however, it already follows from Theorem 3 of the related paper.

\subsection{Zhao and Tang (2011)}

Zhao and Tang [5] analysed the makespan minimization in two-machine no-wait flow shop, where processing times are start time dependent proportional functions and each machine may have non-availability intervals. The authors claimed that the two cases of the problem with an arbitrary number of non-availability intervals are NP-hard in the strong sense (Theorem 3 and Theorem 4 of the related paper). In their proofs they used transformations in which the maximum value of the considered scheduling problems increase in an exponential manner depending on $p$ of 4-P. We only recall the fragments of the transformations (using the notation for 4-P as in [2]), namely, threshold $G=2 t_{0} D^{2 p-1}$ and non-availability intervals $I_{i}=\left(2 t_{0} D^{2 i-1}, t_{0} D^{2 i}\right), 1 \leq i \leq p$ for Theorem 3 and $1 \leq i \leq p-1$ for Theorem 4 . Therefore, the transformations are not pseudopolynomial, but polynomial. Thereby, only NP-hardness of the problems were proved, however, these results are already covered by Theorem 1 and Theorem 2 of the related paper.

\section{Conclusion}

In this note, we showed that the strong NP-hardness proofs of some scheduling problems with start time dependent job processing times that were provided by Gawiejnowicz [2] and Zhao and Tang [5] are incorrect. Therefore, the complete computational status of the analysed problems is still an open issue: ordinary or strongly NP-hard?

Open Access This article is distributed under the terms of the Creative Commons Attribution Noncommercial License which permits any noncommercial use, distribution, and reproduction in any medium, provided the original author(s) and source are credited. 


\section{References}

1. Garey, M.R., Johnson, D.S.: Computers and Intractability: A Guide to the Theory of NP-Completeness. Freeman, San Francisco (1979)

2. Gawiejnowicz, S.: Scheduling deteriorating jobs subject to job or machine availability constraints. Eur. J. Oper. Res. 180, 472-478 (2007)

3. Kononov, A.: Combinatorial complexity of scheduling jobs with simple linear processing times. Diskretny Analiz i Issledovanie Operatsii 3, 15-32 (1996, in Russian)

4. Lee, C.-Y., Vairaktarkis, G. : Complexity of single machine hierarchical scheduling: a survey. In: Pardalos, P.M. (ed.) Complexity in Numerical Optimization, pp. 269-298. World Scientific, Singapore (1993)

5. Zhao, C.L., Tang, H.Y.: A note on two-machine no-wait flow shop scheduling with deteriorating jobs and machine availability constraints. Optim. Lett. 5, 183-190 (2011) 